\title{
Aspectos clínicos e epidemiológicos da conjuntivite alérgiica em serviço de referência
}

\author{
Epidemiological and clinical features of allergic conjunctivitis in \\ a referencecenter
}

\author{
Patricia Maria Fernandes Marback ${ }^{1}$ \\ Denise de Freitas ${ }^{2}$ \\ AugustoParanhos Junior ${ }^{3}$ \\ Rubens Belfort Junior ${ }^{4}$
}

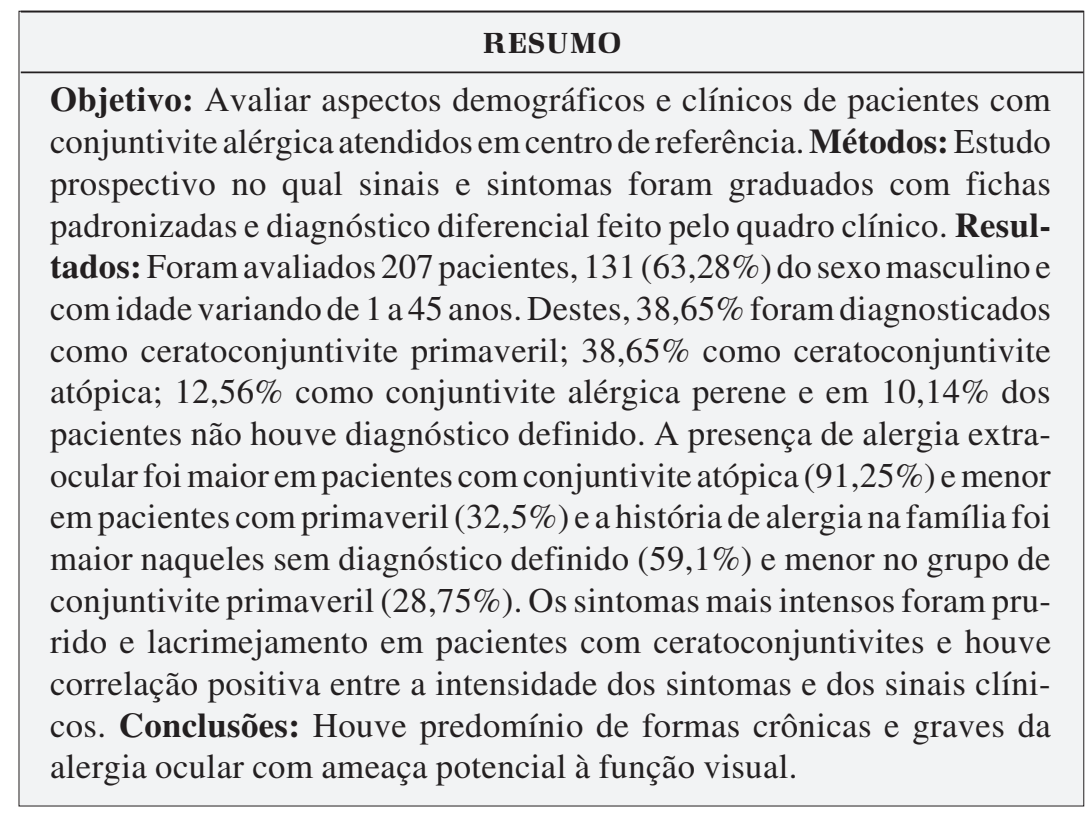

Descritores: Alergia; Conjuntivite alérgica/epidemiologia; Conjuntivite alérgica/diagnóstico
Trabalho realizado no Departamento de Oftalmologia da Universidade Federal de São Paulo - UNIFESP - São Paulo (SP) - Brasil.

Doutora pelo Departamento de Oftalmologia da Universidade Federal de São Paulo - UNIFESP - São Paulo (SP) - Brasil. Médica Assistente do Hospital Universitário Professor Edgard Santos. Faculdade de Medicina, Universidade Federal da Bahia - UFBA - Salvador (BA) Brasil.

${ }^{2}$ Livre Docente do Departamento de Oftalmologia da UNIFESP - São Paulo (SP) - Brasil.

Doutor pelo Departamento de Oftalmologia da UNIFESP - São Paulo (SP) - Brasil.

${ }^{4}$ Professor Titular do Departamento de Oftalmologia da UNIFESP - São Paulo (SP) - Brasil.

Endereço para correspondência: Patricia M. F. Marback. Av. Anita Garibaldi, 1987 - Sala 303 - Centro Médico Albert Sabin. Salvador (BA) CEP 40177-900 E-mail: patriciamarback@uol.com.br

Fonte de auxílio à pesquisa: Fundação Coordenação de Aperfeiçoamento Pessoal de Nível Superior (CAPES).

Recebido para publicação em 30.10.2005

Última versão recebida em 18.08.2006

Aprovação em 20.08.2006

\section{INTRODUÇ̃̃̃O}

Conjuntivite alérgica é definida como uma resposta inflamatória iniciada por reações de hipersensibilidade do tipo I e/ou tipo IV após exposição a um alérgeno ${ }^{(1)}$. A gravidade da reação alérgica se relaciona à intensidade da resposta inflamatória, à idade do paciente e aos fatores genéticos e geográficos $^{(1)}$. O diagnóstico diferencial pode ser feito de acordo com os achados clínicos, embora alguns pacientes, eventualmente, apresentem achados de mais de um tipo de conjuntivite alérgica, resultando em dúvidas diagnósticas $^{(2-3)}$.

Seis tipos de alergia ocular estão descritos na literatura: conjuntivite alérgica sazonal (febre do feno), conjuntivite alérgica perene, ceratoconjuntivite atópica (com a sua variação, blefaroceratoconjuntivite atópica), ceratoconjuntivite primaveril, conjuntivite papilar gigante e dermatite de contato ${ }^{(4)}$.

Existem poucos dados epidemiológicos disponíveis no Brasil sobre alergia ocular. A literatura afirma que a ausência de pólens ou sua baixa concentração em áreas tropicais são insuficientes para causar alergia sazonal (polinose) $)^{(5-6)}$. Mesmo assim, casos isolados foram identificados ${ }^{(7)} \mathrm{em}$ algumas áreas específicas, como Caxias do Sul e Curitiba ${ }^{(8)}$, e a maioria era associada à conjuntivite alérgica sazonal ${ }^{(9)}$. 
Nosso objetivo foi avaliar o padrão clínico e demográfico de pacientes com conjuntivite alérgica atendidos em um serviço de referência em oftalmologia de um centro urbano, caracterizando e quantificando os sinais e sintomas da doença.

\section{MÉTODOS}

Foram estudados, de forma prospectiva, pacientes com conjuntivite alérgica atendidos no Departamento de Oftalmologia da Escola Paulista de Medicina da Universidade Federal de São Paulo. A alergia ocular foi classificada, de acordo com os achados clínicos, em ceratoconjuntivite primaveril (CCP), ceratoconjuntivite atópica (CCA), conjuntivite alérgica sazonal (CAS) e conjuntivite alérgica perene (CAP). Pacientes com conjuntivite papilar gigante (relacionada ao uso de lente de contato ou prótese) e alergia de contato foram excluídos. Pacientes com alergia ocular sem condições de classificação específica foram denominados de não definidos.

Os pacientes foram avaliados através de fichas padronizadas que continham identificação e dados demográficos (idade, sexo, cor, procedência), história clínica (sinais, sintomas, intensidade, duração, períodos de exacerbação e fatores agravantes), alergia extra-ocular, alergia na família. Os sinais e sintomas foram graduados de acordo com a tabela 1.

\section{Análise estatística}

Para comparar a intensidade dos sintomas entre os grupos através dos escores obtidos por cada um, foram usados o teste de Kruskal-Wallis e o teste de comparação múltipla de Dunn. Para observar correlação entre sintomas e comprometimento corneano, foi aplicado o teste de Mann-Whitney.

Para avaliar correlação entre intensidade do quadro clínico com idade de início, duração, sexo, cor da pele, alergia extraocular e alergia na família, foram usados os testes de correlação de Spearman, Man-Whitney e Kruskal-Wallis.

\section{RESULTADOS}

Durante o período estudado, foram examinados 207 pacientes. Destes, $80(38,65 \%)$ tinham CCP; $80(38,65 \%)$, CCA e $26(12,56 \%)$, CAP. Vinte e um pacientes $(10,14 \%)$ foram classificados como não definidos e nenhum paciente foi classificado como sazonal. A tabela 2 mostra os dados de identificação e demográficos dos pacientes.

As percentagens de alergia extra-ocular, alergia na família e fatores agravantes relacionados às diferentes alergias são mostradas na tabela 3. Rinite alérgica foi a associação mais freqüente na CCP $(15,0 \%)$, asma $(55,0 \%)$ e dermatite $(42,5 \%)$ na

\begin{tabular}{|c|c|}
\hline \multicolumn{2}{|c|}{ Tabela 1. Classificação dos sinais e sintomas } \\
\hline Sinais & Sintomas \\
\hline \multirow{16}{*}{$\begin{array}{l}\text { Prurido } \\
\quad 0 \text { - ausente } \\
1 \text { - intermitente } \\
2 \text { - freqüente } \\
3 \text { - muito freqüente } \\
4 \text { - afeta atividades diárias } \\
\text { Sensação de corpo estranho } \\
0 \text { - ausente } \\
1 \text { - sensação de poeira } \\
2 \text { - sensação de areia } \\
3 \text { - sensação de pedra } \\
\text { Fotofobia } \\
0 \text { - ausente } \\
1 \text { - com exposição à luz solar } \\
2 \text { - luz solar ou artificial, melhora com proteção } \\
3 \text { - luz solar ou artificial, não melhora com proteção }\end{array}$} & Papila \\
\hline & 0 - ausente nos $2 / 3$ centrais da conjuntiva tarsal superior \\
\hline & 1 - papilas pequenas nos $2 / 3$ centrais da conjuntiva tarsal superior, sem \\
\hline & espessamento da conjuntiva \\
\hline & 2 - papilas pequenas nos $2 / 3$ centrais da conjuntiva tarsal superior, leve \\
\hline & espessamento da conjuntiva \\
\hline & 3 - espessamento da conjuntiva, reação papilar, dificuldade de ver o padrão \\
\hline & vascular \\
\hline & 4 - papilas gigantes \\
\hline & Inflamação do limbo \\
\hline & 0 - ausente \\
\hline & 1 - edema e hiperemia \\
\hline & 2 - edema, hiperemia e folículos \\
\hline & 3 - edema, hiperemia, folículos, nódulos de Horner-Trantas, ceratite marginal \\
\hline & Ceratite \\
\hline & 0 - ausente \\
\hline \multirow{14}{*}{$\begin{array}{l}\text { Lacrimejamento } \\
\qquad \begin{array}{l}0 \text { - ausente } \\
1 \text { - olhos úmidos } \\
2 \text { - lacrimejamento eventual } \\
3 \text { - lacrimejamento freqüente }\end{array}\end{array}$} & 1 - ponteada fina \\
\hline & 2 - ponteada grosseira, defeito epitelial focal \\
\hline & 3 - úcera em escudo \\
\hline & Hiperemia \\
\hline & 0 - ausente \\
\hline & 1 - aumento do calibre vascular \\
\hline & $\begin{array}{l}2 \text { - leve, mas fácil de detectar } \\
3 \text { - difusa }\end{array}$ \\
\hline & 4 - difusa, mais intensa do que 3 \\
\hline & 5 - olho completamente vermelho \\
\hline & Secreção mucosa \\
\hline & $0-$ ausente \\
\hline & 1 - pequena quantidade em fundo de saco conjuntival \\
\hline & 2 - pequena quantidade em fundo de saco conjuntival, secreção matutina \\
\hline & 3 - olho grudado pela manhã \\
\hline
\end{tabular}




\begin{tabular}{|c|c|c|c|c|}
\hline & $\begin{array}{c}\text { CCP } \\
\text { N (\%) } \\
80(38,6 \%)\end{array}$ & $\begin{array}{c}\text { CCA } \\
\text { N (\%) } \\
80(38,6 \%)\end{array}$ & $\begin{array}{c}\text { CAP } \\
\text { N (\%) } \\
26(10,1 \%)\end{array}$ & $\begin{array}{c}\text { Não definido } \\
N(\%) \\
21(12,6 \%)\end{array}$ \\
\hline Masculino & $69(86,3 \%)$ & $42(52,5 \%)$ & $7(26,9 \%)$ & $13(61,9 \%)$ \\
\hline Idade (anos) & $9,1 \pm 4,5$ & $14,6 \pm 7,0$ & $15,3 \pm 10,4$ & $9,4 \pm 6,3$ \\
\hline Branco & $17(21,3 \%)$ & $16(20,0 \%)$ & $12(46,2 \%)$ & $5(23,8 \%)$ \\
\hline Negro & $9(11,3 \%)$ & $10(12,5 \%)$ & $3(11,5 \%)$ & $3(14,3 \%)$ \\
\hline Mulato & $53(66,3 \%)$ & $54(67,5 \%)$ & $11(42,3 \%)$ & $13(61,9 \%)$ \\
\hline Amarelo & $1(1,3 \%)$ & - & - & - \\
\hline
\end{tabular}

CCA e rinite alérgica $(69,2 \%)$ na CAP. O grupo de diagnóstico não definido teve asma como a alergia extra-ocular mais freqüente $(59,1 \%)$. Em todos os grupos houve relato de alergia na família, sendo que o grupo CCP teve uma percentagem menor $(28,7 \%)$. Fatores agravantes como frio, calor, poeira, alimentos e outros (incluindo cosméticos, produtos de limpeza, pelo de animais) foram mencionados em percentagens variadas como fatores precipitantes ou agravantes do quadro clínico ocular. Todos os pacientes apresentavam sintomas perenes e nenhum deles mencionou pólen como um fator precipitante ou agravante (Tabela 3 ).

Acuidade visual foi pior do que $20 / 50 \mathrm{em}$ pelo menos um dos olhos em $14,08 \%$ dos pacientes com primaveril e em $31,64 \%$ dos pacientes com atópica.

A tabela 4 mostra que o prurido foi mais grave em pacientes com CCP e CCA $(\mathrm{p}<0,05)$ e o lacrimejamento foi maior em pacientes com primaveril $(\mathrm{p}<0,05)$ (Teste de comparação múltipla de Dunn). Houve correlação estatística entre a intensidade de cada sintoma e a presença de ceratite (Mann-Whitney, $\mathrm{p}<0,05$ ).

A correlação entre intensidade de sinais e sintomas e ceratite em pacientes com ceratoconjuntivite primaveril, ceratoconjuntivite atópica e conjuntivite alérgica perene é mostrada

\begin{tabular}{|c|c|c|c|c|}
\hline & $\begin{array}{c}\mathrm{CCP} \\
80\end{array}$ & $\begin{array}{c}\text { CCA } \\
80\end{array}$ & $\begin{array}{l}\text { Não definido } \\
21\end{array}$ & $\begin{array}{l}\text { CAP } \\
26\end{array}$ \\
\hline Alergia extra-ocular & $32,5 \%$ & $91,2 \%$ & $72,7 \%$ & $88,5 \%$ \\
\hline Asma & $13,7 \%$ & $55,0 \%$ & $59,1 \%$ & $30,8 \%$ \\
\hline Rinite & $15,0 \%$ & $41,9 \%$ & $31,8 \%$ & $69,2 \%$ \\
\hline Dermatite & $3,7 \%$ & $42,5 \%$ & $22,7 \%$ & - \\
\hline Outros & $5,0 \%$ & $7,5 \%$ & $9,1 \%$ & $19,2 \%$ \\
\hline Alergia na família & $28,7 \%$ & $55,0 \%$ & $59,1 \%$ & $42,3 \%$ \\
\hline Fatores agravantes & $88,7 \%$ & $76,2 \%$ & $68,2 \%$ & $67,7 \%$ \\
\hline Frio & $2,5 \%$ & $13,7 \%$ & $9,1 \%$ & $15,4 \%$ \\
\hline Calor & $68,7 \%$ & $37,5 \%$ & $40,9 \%$ & $26,9 \%$ \\
\hline Poeira doméstica & $52,5 \%$ & $53,7 \%$ & $36,4 \%$ & $34,6 \%$ \\
\hline Pólen & 0 & 0 & 0 & 0 \\
\hline Alimento & $6,2 \%$ & $12,5 \%$ & $27,7 \%$ & - \\
\hline Outros & $6,2 \%$ & $7,5 \%$ & $13,6 \%$ & - \\
\hline \multicolumn{5}{|c|}{$\begin{array}{l}\mathrm{CCP}=\text { ceratoconjuntivite primaveril; } \mathrm{CCA}=\text { ceratoconjuntivite atópica; } \mathrm{CAP}= \\
\text { conjuntivite alérgica perene }\end{array}$} \\
\hline
\end{tabular}

na tabela 5. Nela, observamos que fotofobia, sensação de corpo estranho, lacrimejamento e prurido eram significativamente mais intensos quando graduados por pacientes que apresentavam ceratite no momento da consulta do que por aqueles que não apresentavam. Em pacientes com CCA, a intensidade dos sinais e sintomas foi maior naqueles com alergia extra-ocular (Tabela 5). Pacientes do sexo masculino com CCA tiveram achados clínicos mais intensos do que pacientes do sexo feminino.

Foram correlacionados dados demográficos (idade, sexo, cor da pele) com a intensidade de sinais e de sintomas de cada grupo e com a presença de alergia extra-ocular e história de alergia na família. Na tabela 6 mostramos os resultados estatisticamente significantes $(\mathrm{p}<0,05)$, sendo que houve correlação positiva entre o escore dos sinais e dos sintomas $(\mathrm{p}=0,00)$. Observamos que pacientes com CCP e história de alergia na

Tabela 4. Intensidade dos sintomas no grupo estudado

\begin{tabular}{|c|c|c|}
\hline Sintomas & $\mathbf{p}^{*}$ & $(p<0,05)^{\star \star}$ \\
\hline \multirow[t]{2}{*}{ Prurido } & 0,0010 & $\mathrm{CCA}>\mathrm{CAP}$ \\
\hline & & $\mathrm{CCP}>\mathrm{CAP}$ \\
\hline Sensação de corpo estranho & 0,6693 & - \\
\hline Lacrimejamento & 0,0071 & $\mathrm{CCP}>\mathrm{CAP}$ \\
\hline Fotofobia & 0,0719 & - \\
\hline \multicolumn{3}{|c|}{$\begin{array}{l}{ }^{*} \text { Kruskal-Wallis } \\
{ }^{*} \text { Dunn (teste de comparação múltipla) } \\
\text { CCA= ceratococnjuntivite atópica; } \mathrm{CAP}=\text { conjuntivite alérgica perene; } \mathrm{CCP}= \\
\text { ceratoconjuntivite primaveril }\end{array}$} \\
\hline
\end{tabular}

Tabela 5. Intensidade dos sintomas e presença de ceratite. Escore do prurido variando de 0 a 4 , sensação de corpo estranho de 0 a 3 , lacrimejamento de 0 a 3 e fotofobia de 0 a 3

\begin{tabular}{|c|c|c|c|c|c|}
\hline & \multicolumn{2}{|c|}{$\begin{array}{l}\text { Ceratite } \\
(n=94)\end{array}$} & \multicolumn{2}{|c|}{$\begin{array}{l}\text { Sem ceratite } \\
\quad(n=113)\end{array}$} & \multirow[t]{2}{*}{$p$} \\
\hline & QI & QS & QI & QS & \\
\hline Prurido (0 a 4) & 2,75 & 4 & 2 & 3 & $<0,0001^{*}$ \\
\hline $\begin{array}{l}\text { Sensação de corpo } \\
\text { estranho }(0 \text { a } 3)\end{array}$ & 0 & 2 & 0 & 1 & $0,0080^{*}$ \\
\hline Fotofobia (0 a 3) & 1 & 2 & 0 & 1 & $<0,0001^{*}$ \\
\hline Lacrimejamento (0 a 3) & 1 & 3 & 0 & 3 & $<0,0001^{\star}$ \\
\hline $\begin{array}{l}{ }^{*} \text { Mann-Whitney, } \mathrm{p}<0,05 \\
\mathrm{Ql}=\text { quartil inferior; } \mathrm{QS}=\mathrm{q}\end{array}$ & I su & & & & \\
\hline
\end{tabular}




\begin{tabular}{|c|c|c|c|c|c|c|c|c|}
\hline \multirow[t]{2}{*}{ Diagnóstico } & \multirow{2}{*}{\multicolumn{2}{|c|}{${ }^{* * *} p=0,00$}} & \multicolumn{3}{|c|}{ Sintomas } & \multicolumn{3}{|c|}{ Sinais } \\
\hline & & & QI & QS & $p$ & QI & QS & $p$ \\
\hline \multirow[t]{2}{*}{ CAP } & Sexo & Masculino & 2,25 & 4,75 & \multirow{2}{*}{$0,0164^{\star}$} & 2 & 2 & \multirow{2}{*}{$0,6013^{*}$} \\
\hline & & Feminino & 4 & 8,75 & & 2 & 3 & \\
\hline \multirow[t]{2}{*}{ CCA } & Sexo & Masculino & 5 & 9,75 & \multirow{2}{*}{$0,6329^{*}$} & 4 & 9 & \multirow{2}{*}{$0,0054^{*}$} \\
\hline & & Feminino & 5 & 11,25 & & 3 & 6,25 & \\
\hline \multirow[t]{2}{*}{$\mathrm{CCA}$} & Extra-ocular & Sim & 5 & 11 & \multirow{2}{*}{$0,0485^{\star *}$} & 4 & 11 & \multirow{2}{*}{$0,4089^{* *}$} \\
\hline & & Não & 3,25 & 9,5 & & 3,25 & 10 & \\
\hline \multirow[t]{2}{*}{ CCP } & Alergia família & Sim & 4 & 11,3 & \multirow{2}{*}{$0,2553^{\star *}$} & 5 & 9 & \multirow{2}{*}{$0,0498^{\star \star}$} \\
\hline & & Não & 8 & 12 & & 4 & 8 & \\
\hline
\end{tabular}

família obtiveram maior escore de sinais ( $\mathrm{p}=0,0498)$, mas não de sintomas $(\mathrm{p}=0,2553)$. No grupo com CCA, os sintomas foram mais intensos naqueles com alergia extra-ocular ( $\mathrm{p}=0,0438)$, e o escore de sinais foi maior em pacientes do sexo masculino $(\mathrm{p}=0,0054)$. Pacientes do sexo feminino com CAP tiveram sintomas mais intensos do que pacientes do sexo masculino com o mesmo diagnóstico ( $\mathrm{p}=0,0164)$, o que não ocorreu com os sinais $(\mathrm{p}=0,6013)$.

\section{DISCUSS ÃO}

Avaliamos nesta série 207 pacientes com diagnóstico clínico de alergia ocular. A classificação precisa não foi possível em $10,14 \%$ dos pacientes, pois estes apresentavam achados que se imbricavam tanto no grupo de CCP quanto no grupo de CCA. Alguns autores discutem as dificuldades no diagnóstico diferencial da alergia ocular, e a literatura revisada mostrou que um mesmo termo é usado para nomear diferentes diagnósticos e um mesmo diagnóstico pode ser classificado com termos diferentes $^{(10)}$. Em uma série de 120 pacientes referidos com diagnóstico de primaveril, 32 foram excluídos devido a erro diagnóstico ${ }^{(11)}$.

Nenhum dos nossos pacientes foi diagnosticado como conjuntivite alérgica sazonal. Goulart et al. encontraram apenas $18,6 \%$ em 176 pacientes com alergia ocular ${ }^{(12)}$. A baixa frequiência de febre do feno em várias localidades brasileiras pode ser explicada pela falta de susceptibilidade em nossa população, baixa densidade de pólen nas estações, estação polínica muito curta ou pólens com baixo poder antigênico nos territórios estudados ${ }^{(5)}$. Outra possibilidade é que pessoas que vivem em áreas urbanas podem apresentar maior tendência a doenças alérgicas perenes, devido à exposição crônica a poeira, ácaros e fungos em locais com maior umidade. Além do mais, estudos mostram que a prevalência de doença alérgica (asma e polinose) tende a ser maior em centros urbanos do que em áreas rurais ${ }^{(13)}$.

A idade dos pacientes na data de inclusão no estudo foi maior naqueles com diagnóstico de CCA do que os que tinham diagnóstico de CCP, mas muitos dos pacientes com CCA tinham idade inferior a 14 anos. Embora existam pacientes jovens em algumas publicações sobre CCA, a maioria dos autores caracteriza a ceratoconjuntivite atópica como uma doença de aparecimento mais tardio, em idade adulta, iniciada no final da adolescência ${ }^{(2,14-15)}$.

Trinta e dois e meio por cento dos pacientes com CCP relataram alergia extra-ocular, e 28,75\%, história de alergia na família. Na literatura, as frequiências das alergias são variáveis. Neumann et al. encontraram $11 \%$ de alergia extra-ocular e $15 \%$ de história de alergia na família nos seus casos de $\mathrm{CCP}^{(16)}$. Outros autores acreditam que a maioria dos pacientes com CCP tem algum outro tipo de alergia ${ }^{(17-18)}$. Na Itália, encontraram $40 \%$ dos pacientes com primaveril apresentando outra forma de alergia associada ${ }^{(19)}$. Em série retrospectiva publicada, 41,5\% dos pacientes com CCP tinham doença sistêmica atópica e 48,7\% tinham antecedente de alergia na família ${ }^{(20)}$. Em um estudo de cohort de 62 pacientes na Suécia, a freqüência de alergia extra-ocular em pacientes com CCP variou de acordo com o grupo étnico de $8,3 \%$ em asiáticos a $100 \%$ em latino-americanos. Em 109 pacientes consecutivos avaliados em um período de dois anos na Nigéria, apenas 4,5\% apresentavam alergia extra-ocular ${ }^{(21)}$. No Brasil, estudos encontraram $76 \%$ de positividade de teste cutâneo para hipersensibilidade imediata (Prick test) em pacientes com $\mathrm{CCP}^{(22)}$.

Poeira doméstica e temperaturas altas foram os fatores precipitantes ou agravantes mais freqüentes em todo o nosso grupo estudado. A piora dos sintomas associada a algum tipo de alimento foi mais freqüente no grupo de atópica e de diagnóstico indefinido. Reações de hipersensibilidade a alimentos, causando manifestações fora do trato gastro-intestinal já são conhecidas $^{(23)}$, geralmente em crianças com dermatite atópica ${ }^{(24)}$.

Segundo estudo realizado em 1991, pacientes com CCA apresentam piora dos sintomas na presença de poeira em $43 \%$ dos casos, com alimentos em $35 \%$ dos casos e neste estudo não houve menção a pólen ${ }^{(3)}$. Outros autores mostraram que 52,2\% dos pacientes com diagnóstico de CCP apresentavam teste cutâneo de hipersensibilidade imediata positivo, sendo $71,6 \%$ sensibilizados tanto para antígenos perenes (Dermatophagoides pteronissinus), quanto para antígenos sazonais (gramíneas) ${ }^{(20)}$.

$\mathrm{O}$ grupo de CCA apresentou pacientes com acuidade visual pior do que 20/200 no melhor olho em 1,27\% dos casos 
pior do que 20/50 em 31,64\% em pelo menos um dos olhos. Causas de baixa visão em alguns destes pacientes incluem ectasia corneana, cicatriz corneana, úlcera em escudo, ceratite infecciosa entre outras ${ }^{(25)}$. Pacientes com ceratoconjuntivite primaveril apresentam complicações corneanas e límbicas, como cicatrizes, astigmatismo irregular, hiperplasia da conjuntiva limbar e ceratocone ${ }^{(26-27)}$. O astigmatismo irregular apresentado pelos pacientes pode estar relacionado às alterações epiteliais que ocorrem durante o período de agudização das ceratoconjuntivites, tanto primaveril quanto atópica e podem regredir após o tratamento ${ }^{(28)}$.

\section{CONCLUSÕES}

Houve predomínio do sexo masculino, de cor da pele parda e de formas graves e crônicas. Dos sintomas, o prurido ocular foi o mais prevalente e o comprometimento da acuidade visual foi maior em pacientes com ceratoconjuntivite atópica. Esta foi uma série estudada em um serviço de referência. Apesar do viés relacionado a esta condição, a maioria dos pacientes eram crianças com uma doença que representa ameaça potencial à função visual. A dificuldade de coleta de dados em crianças é outro fato que deve ser enfatizado neste estudo. A ajuda dos pais ou responsáveis na gradação dos sintomas aumenta a subjetividade das respostas, mas o exame oftalmológico com a graduação dos sinais, e sua correlação com os sintomas, são dados objetivos que, neste grupo, nos mostram o predomínio de formas graves da doença.

\section{ABSTRACT}

Background: To evaluate demographic and clinical features of patients with allergic conjunctivitis in a reference center. Methods: Prospective study using sings and symptoms graduated by standardized charts for clinical diagnosis. Results: We evaluated 207 patients, age ranging form 1 to 45 years and of whom $131(63.28 \%)$ were males. Of the patients, $38.65 \%$ presented vernal keratoconjunctivitis; $38.65 \%$, atopic keratoconjunctivitis; $12.56 \%$, perennial allergic conjunctivitis and in $10.14 \%$ patients the diagnosis was not defined. Extraocular allergy was more frequent in patients with atopic keratoconjunctivitis $(91.25 \%)$ and less frequent in patients with vernal keratoconjunctivitis (32.5\%). Family history of allergy was more frequent in patients without defined diagnosis $(59.1 \%)$ and less frequent in the vernal group (28.75\%). The most itense symptoms were itching and tearing in patients with keratoconjunctivitis. There was a positive correlation between symptom intensity and signs severity. Conclusion: Chronic and severe types of ocular allergy, with potential threat to visual function, predominated in the studed group.

Keywords: Allergy; Conjunctivitis, allergic/epidemiology; Conjunctivitis, allergic/diagnosis

\section{REFERÊNCIAS}

1. Nishiwaki-Dantas MC, Finzy S. Conjuntivites Alérgicas. In: Lima ALH, Nishiwaki-Dantas MC, Alves MR, editores. Doenças externas oculares e córnea Manual do CBO;. Reimpressão - Rio de Janeiro: Cultura Médica; São Paulo: CBO, 2006. p. 176-86.

2. Marback PMF, Freitas D, Belfort Júnior R. Alergia ocular estratégias de tratamento. Rev Bras Oftalmol. 2001;60(1):10-2.

3. Tuft SJ, Kemeny DM, Dart JK, Buckley RJ. Clinical features of atopic keratoconjunctivitis. Ophthalmology. 1991;98(2):150-8.

4. Friedlaender MH. Conjunctivitis of allergic origin: clinical presentation and differential diagnosis. Surv Ophthalmol. 1993;38(Suppl):105-14.

5. Lima AO, Dias da Costa P, Galeno R, Santos PP. Pollinosis in Brazil. Ann Allergy. 1946;4:13-7

6. Salles VLFF, Kase MT, Adissi M, Mori JC, Mello JF. Contagem de pólens aéreos no município de São Paulo. Rev Bras Alergia Imunopatol. 1988;11(3): 79-84.

7. Vieira FAM. Polinose no Brasil. In: Negreiros B, Ungier C. Alergologia clínica. São Paulo: Atheneu; 1995. p. 106-11.

8. Vieira FAM, Negreiros EB. Epidemiologia da polinose na população de algumas cidades do Estado do Rio Grande do Sul. Rev Bras Alergia Imunopatol. 1989;12(3):73-8.

9. Rosário Filho NA. Reflexões sobre polinose: 20 anos de experiência. Rev Bras Alergia Imunopatol. 1997;20(6):210-3.

10. Calonge M. Classification of ocular atopic/allergic disorders and conditions: an unsolved problem. Acta Ophthalmol Scand Suppl. 1999;(228):10-3.

11. Caldwell DR, Verin P, Hartwich-Young R, Meyer SM, Drake MM. Efficacy and safety of lodoxamide $0.1 \%$ vs cromolyn sodium $4 \%$ in patients with vernal keratoconjunctivitis. Am J Ophthalmol. 1992;113(6):632-7.

12. Goulart DA, Goulart DG, Cypel MC, Dantas PEC, Nishiwaki-Dantas MC. Perfil clínico-epidemiológico de pacientes do Ambulatório de Alergia Ocular da Santa Casa de São Paulo. Arq Bras Oftalmol. 2003;66(5):609-15.

13. Burr ML, Emberlin JC, Treu R, Cheng S, Pearce NE. ISAAC Phase One Study Group. Pollen counts in relation to prevalence of allergic rhinoconjunctivitis, asthma and atopic eczema in the International Study of Asthma and Allergies in Childhood (ISAAC). Clin Exp Allergy. 2003;33(12):1675-80.

14. Foster CS, Calonge M. Atopic keratoconjunctivitis. Ophthalmology. 1990;97 (8):992-1000.

15. Power WJ, Tugal-Tutkun I, Foster CS. Long-term follow-up of patients with atopic keratoconjunctivitis. Ophthalmology. 1998;105(4):637-42.

16. Neumann E, Gutmann MJ, Blumenkrantz N, Michaelson IC. A review of four hundred cases of vernal conjunctivitis. Am J Ophthalmol. 1959;47(2):166-72.

17. Buckley RJ. Vernal keratoconjunctivitis. Int Ophthalmol Clin. 1988;28(4): 303-8.

18. Friedlaender MH, Cameron J. Vernal keratoconjunctivitis and trachoma. Int Ophthalmol. 1988;12(1):47-51.

19. Leonardi A, Borghesan F, Faggian D, Depaoli M, Secchi AG, Plebani M. Tear and serum soluble leukocyte activation markers in conjunctival allergic diseases. Am J Ophthalmol. 2000;129(2):151-8.

20. Bonini S, Bonini S, Lambiase A, Marchi S, Pasqualetti P, Zuccaro O, et al. Vernal keratoconjunctivitis revisited: a case series of 195 patients with longterm follow-up. Ophthalmology. 2000;107(6):1157-63.

21. Ukponmwan CU. Vernal keratoconjunctivitis in Nigerians: 109 consecutive cases. Trop Doct. 2003;33(4):242-5.

22. Goulart DA, Schneider DM, Tanaka E, Santos MCM, Nishiwaki-Dantas MC, Forte WCN. Participação da sensibilidade atópica em pacientes com ceratoconjuntivite alérgica primaveril. Arq Bras Oftalmol. 2004;67(3):411-4.

23. Ortega Cisneros M, Vidales Díaz MA, del Río Navarro BE, Sienra Monge JJL. Reactividad cutánea a alimentos en pacientes con rinoconjuntivitis alérgica. Alergia Méx. 1997;44(6):153-7.

24. Maldonado AM. Alergia alimentaria y eccema. Arch Argent Alerg Inmunol Clín. 1994;25(3):19-22.

25. Botelho PBM, Marback P, Sousa LB, Campos M, Vieira LA. Ceratoconjuntivite alérgica e complicações no segmento ocular anterior de pacientes. Arq Bras Oftalmol. 2003;66(1):25-8.

26. Tabbara KF, Butrus SI. Vernal keratoconjunctivitis and keratoconus. Am J Ophthalmol. 1983;95(5):704-5.

27. Tabbara KF. Ocular complications of vernal keratoconjunctivitis. Can J Ophthalmol. 1999;34(2):88-92.

28. Secchi AG, Leonard A, Tognon MS, Ciuccio N. Astigmatic changes in limbal vernal keratoconjunctivitis [abstract]. Invest Ophthalmol Vis Sci. 1992;33 (Suppl):994. 